\title{
Quark stars under strong magnetic fields
}

\author{
Peng-Cheng Chu, ${ }^{1}$ Lie-Wen Chen*, ${ }^{1,2}$ and Xin Wang ${ }^{1}$ \\ ${ }^{1}$ Department of Physics and Astronomy and Shanghai Key Laboratory for Particle Physics and Cosmology, \\ Shanghai Jiao Tong University, Shanghai 200240, China \\ ${ }^{2}$ Center of Theoretical Nuclear Physics, National Laboratory of Heavy Ion Accelerator, Lanzhou 730000, China
}

(Dated: March 22, 2022)

\begin{abstract}
Within the confined isospin- and density-dependent mass model, we study the properties of strange quark matter (SQM) and quark stars (QSs) under strong magnetic fields. The equation of state of SQM under a constant magnetic field is obtained self-consistently and the pressure perpendicular to the magnetic field is shown to be larger than that parallel to the magnetic field, implying that the properties of magnetized QSs generally depend on both the strength and the orientation of the magnetic fields distributed inside the stars. Using a density-dependent magnetic field profile which is introduced to mimic the magnetic field strength distribution in a star, we study the properties of static spherical QSs by assuming two extreme cases for the magnetic field orientation in the stars, i.e., the radial orientation in which the local magnetic fields are along the radial direction and the transverse orientation in which the local magnetic fields are randomly oriented but perpendicular to the radial direction. Our results indicate that including the magnetic fields with radial (transverse) orientation can significantly decrease (increase) the maximum mass of QSs, demonstrating the importance of the magnetic field orientation inside the magnetized compact stars.
\end{abstract}

PACS numbers: 21.65.Qr, 97.60.Jd, 26.60.Kp

\section{INTRODUCTION}

The compact stars provide a unique astrophysical testing ground to explore the nature of matter under extreme conditions [1, 2]. Neutron stars (NSs) are a class of densest compact stars in the universe. In the interior of NSs, the baryon number density can reach or even be larger than about 6 times normal nuclear matter density and thus hyperons, meson condensations and even quark matter may be present there. The study of NSs has provided us important information about the equation of state (EOS) of neutron-rich nuclear matter [3, 4]. Theoretically, NSs may be converted to (strange) quark stars (QSs), which is made purely of deconfined $u, d$, and $s$ quark matter (with some leptons due to charge neutrality and $\beta$-equilibrium), i.e., strange quark matter (SQM) [5 7]. The possible existence of QSs is one of the most intriguing aspects of modern astrophysics and has important implications for the strong interaction matter at high baryon densities, especially the properties of SQM that essentially determine the structure of QSs. In terrestrial laboratories, the properties of SQM can be explored by heavy ion collisions, e.g., the beamenergy scan program at RHIC as well as the experiments planned in the Facility for Antiproton and Ion Research (FAIR) at GSI and the Nuclotron-based Ion Collider Facility (NICA) at JINR, which aim to give a detailed picture of the QCD phase structure, especially to locate the so-called QCD critical point [8]. These studies on SQM have become nowadays one of the fundamental issues in nuclear physics, astrophysics and cosmology.

\footnotetext{
${ }^{*}$ Corresponding author (email: lwchen@sjtu.edu.cn)
}

Theoretically, according to the Bodmer-WittenTerazawa hypothesis [9 11], SQM might be the true ground state of QCD matter (i.e., the strong interaction matter) and is absolutely stable. The properties of SQM in QSs cannot be calculated directly by either the $a b$ initio Lattice QCD or the perturbative QCD (pQCD) because of the difficulty in treating the finite baryon chemical potential or the low energy scale, and thus a number of phenomenological models have been proposed to explore the properties of SQM, such as MIT bag model [12 16], the Nambu-Jona-Lasinio (NJL) model [17 20], the pQCD approach [21 24], the Dyson-Schwinger approach [25 27], the confined-densitydependent-mass (CDDM) model 28 35], and the quasiparticle model 36, 37]. Within an isospin-extended version of the CDDM model, i.e., the confined isospin- and density-dependent mass (CIDDM) model 38] in which the quark confinement is modeled by the density- and isospin-dependent quark masses, it has been shown recently that QSs provide an excellent astrophysical laboratory to explore the properties of SQM, especially the quark matter symmetry energy.

In the work of Ref. 38], it has been assumed that the magnetic field effects can be neglected in QSs. An important aspect of the compact star physics is that compact stars could be endowed with strong magnetic fields. Large magnetic field strength of $B \sim 10^{14} \mathrm{G}$ has been estimated at the surface of compact stars 39 41]. The magnetic field strength may reach as large as $B \sim 10^{18}$ $\mathrm{G}$ in the core of compact stars [42]. In the work by Ferrer et al. [43], the estimated magnetic field strength in the core of the self-bound QSs can even reach about $10^{20}$ G. In such tremendous magnetic fields, the spatial rotational $(\mathcal{O}(3))$ symmetry will break and one must consider the pressure anisotropy of the system [43 46]. Fur- 
thermore, in order to describe the spatial distribution of the magnetic field strength in compact stars, people usually introduce a density-dependent magnetic field profile [47, 48]. Therefore, it is interesting and important to study the effects of the spatial distribution of the magnetic field strength and orientation on the properties of compact stars. These studies are critical for accurately determining the properties (e.g., EOS) of dense matter by comparing the model results with the astrophysical observations of compact stars. As a matter of fact, it is still controversial about if the inclusion of the magnetic fields can enhance or reduce the maximum mass of the compact stars [47 [57]. The main motivation of the present work is to explore the properties of SQM and QSs under strong magnetic fields. We demonstrate that both the strength distribution and the orientation of the magnetic fields inside the QSs are important for understanding the properties of the QSs, and depending on the magnetic field orientation, the maximum QS mass can be either enhanced or reduced.

The paper is organized as follows. We describe in Sec. III the theoretical models and methods used in the present paper, and then present the results and discussions in Sec. III. Finally, a conclusion is given in Sec. IV

\section{MODELS AND METHODS}

\section{A. The confined isospin- and density-dependent mass model}

The confined isospin- and density-dependent mass (i.e., the CIDDM) model 38] is an extended version of the CDDM model 28 35. for quark matter by introducing the isospin dependence of the quark equivalent mass. In the CIDDM model, the quark confinement is modeled by the density- and isospin-dependent quark masses. Particularly, the equivalent quark mass in isospin asymmetric quark matter with baryon number density $n_{B}$ and isospin asymmetry $\delta$ is expressed as

$$
\begin{aligned}
m_{q} & =m_{q_{0}}+m_{I}+m_{i s o} \\
& =m_{q_{0}}+\frac{D}{n_{B}^{z}}-\tau_{q} \delta D_{I} n_{B}^{\alpha} e^{-\beta n_{B}}
\end{aligned}
$$

where $m_{q 0}$ is the quark current mass, $m_{I}=\frac{D}{n_{B} z}$ reflects the flavor-independent quark interactions in quark matter, and $m_{\text {iso }}=-\tau_{q} \delta D_{I} n_{B}^{\alpha} e^{-\beta n_{B}}$ represents the isospin dependent quark interactions in quark matter. For $m_{I}=$ $\frac{D}{n_{B} z}$, the constant $z$ is the quark mass scaling parameter and the constant $D$ is a parameter determined by stability arguments of SQM. For $m_{\text {iso }}=-\tau_{q} \delta D_{I} n_{B}^{\alpha} e^{-\beta n_{B}}$, the constants $D_{I}, \alpha$ and $\beta$ are parameters determining the isospin dependence of quark effective interactions in quark matter, $\tau_{q}$ is the isospin quantum number for quarks and we set $\tau_{q}=1$ for $q=u$ ( $u$ quarks), $\tau_{q}=-1$ for $q=d$ ( $d$ quarks), and $\tau_{q}=0$ for $q=s$ ( $s$ quarks). As usual [58 61], the isospin asymmetry is defined as

$$
\delta=3 \frac{n_{d}-n_{u}}{n_{d}+n_{u}}
$$

which equals to $-n_{3} / n_{B}$ with the isospin density $n_{3}=$ $n_{u}-n_{d}$ and $n_{B}=\left(n_{u}+n_{d}\right) / 3$ for two-flavor $u$ - $d$ quark matter. Especially, one has $\delta=1(-1)$ for quark matter converted by pure neutron (proton) matter according to the nucleon constituent quark structure, consistent with the conventional definition for nuclear matter, i.e., $\frac{n_{n}-n_{p}}{n_{n}+n_{p}}=-n_{3} / n_{B}$

From Eq. (1), one can see that the quark confinement condition $\lim _{n_{B} \rightarrow 0} m_{q}=\infty$ can be guaranteed if $z>0$ and $\alpha \geq 0$. Furthermore, if $\beta>0$, one then has $\lim _{n_{B} \rightarrow \infty} m_{\text {iso }}=0$ and thus the asymptotic freedom $\lim _{n_{B} \rightarrow \infty} m_{q}=m_{q 0}$ is satisfied. For two-flavor $u$ - $d$ quark matter, the chiral symmetry is restored at high density due to $\lim _{n_{B} \rightarrow \infty} m_{q}=0$ if the current masses of $u$ and $d$ quarks are neglected. In addition, the equivalent quark mass in Eq. (1) also satisfies the exchange symmetry between $u$ and $d$ quarks which is required by isospin symmetry of the strong interaction. Therefore, the phenomenological parametrization form of the isospin dependent equivalent quark mass in Eq. (1) is quite general and respects the basic features of QCD. For more details about the CIDDM model, the reader is referred to Ref. [38].

As demonstrated in Ref. 38], including the isospin dependent quark effective interactions $m_{\text {iso }}$ in the equivalent quark mass can significantly influence the quark matter symmetry energy as well as the properties of SQM and QSs. In particular, the most recently discovered large mass pulsar PSR J0348+0432 with a mass of $2.01 \pm 0.04 M_{\odot}$ can be described as a QS within the CIDDM model if the two-flavor $u$ - $d$ quark matter symmetry energy is large enough and the value of the quark mass scaling parameter $z$ is selected appropriately. For instance, the parameter set DI-85 $(z=1.8)$, for which we have $D_{I}=85 \mathrm{MeV} \cdot \mathrm{fm}^{3 \alpha}, \alpha=0.7, \beta=0.1 \mathrm{fm}^{3}$, $D=22.922 \mathrm{MeV} \cdot \mathrm{fm}^{-3 z}, z=1.8, m_{u 0}=m_{d 0}=5.5 \mathrm{MeV}$ and $m_{s 0}=80 \mathrm{MeV}$, can predict a QS with a mass of $2.01 M_{\odot}$, corresponding to the measured center value for the pulsar PSR J0348+0432 62]. The corresponding radius of the predicted QS (with a mass of $2.01 M_{\odot}$ ) is 9.98 $\mathrm{km}$, the central baryon number density is $1.25 \mathrm{fm}^{-3}$, and the surface (zero-pressure point) baryon number density is $0.465 \mathrm{fm}^{-3}$. In addition, the strength of the two-flavor $u-d$ quark matter symmetry energy from DI-85 $(z=1.8)$ is about two times larger than that of the free quark gas or that predicted by the conventional NJL model. In this work, we study the properties of SQM and QS's under strong magnetic fields in the CIDDM model with DI-85 $(z=1.8)$. 


\section{B. SQM under a constant magnetic field}

The energy spectrum of a fermion (e.g., quarks and leptons) with electric charge $q_{i}$ in an external constant magnetic field with strength $B$ can be expressed as 63 .

$$
E_{p, i}=\sqrt{p_{z}^{2}+2 \nu\left|q_{i}\right| B+m_{i}^{2}}
$$

where $p_{z}$ is the momentum in the $z$ direction (here the magnetic field is assumed to be along the $z$ axis), $m_{i}$ is mass, and $\nu=n+\frac{1}{2}-\frac{q_{i}}{\left|q_{i}\right|} \frac{s}{2}$ represents the Landau levels with $n=0,1,2,3, \ldots$ being the principal quantum number and $s= \pm 1$ denoting the spin ("+1" for spinup and " -1 " for spin-down). Similarly to the works in Refs. [46 48, 64 66], we do not consider here the contributions from the anomalous magnetic moments since they are not well understood for quarks in the deconfined condition and are insignificant for leptons 67.

In this work, we study the thermodynamic properties of magnetized SQM at zero temperature within the CIDDM model. The thermodynamic potential of SQM under a constant magnetic field $B$ can then be expressed as

$$
\Omega=\sum_{i=u, d, s, l} \Omega_{i}
$$

with

$$
\begin{aligned}
\Omega_{i}= & -\sum_{\nu=0}^{\nu_{\max }^{i}} \frac{g_{i}\left(\left|q_{i}\right| B\right)}{4 \pi^{2}} \alpha_{\nu} \int_{-\infty}^{\infty} \mathrm{d} p_{z}\left[\mu_{i}^{*}-E_{p, i}\right] \\
= & -\sum_{\nu=0}^{\nu_{\max }^{i}} \frac{g_{i}\left(\left|q_{i}\right| B\right)}{2 \pi^{2}} \alpha_{\nu}\left\{\frac{1}{2} \mu_{i}^{*} \sqrt{\mu_{i}^{* 2}-s_{i}(\nu, B)^{2}}\right. \\
& \left.-\frac{s_{i}(\nu, B)^{2}}{2} \ln \left[\frac{\mu_{i}^{*}+\sqrt{\mu_{i}^{* 2}-s_{i}(\nu, B)^{2}}}{s_{i}(\nu, B)}\right]\right\} .
\end{aligned}
$$

In the above expressions, $\Omega_{i}$ represents each particle contribution to the thermodynamic potential, $i$ in the sum is for all flavors of quarks and leptons, and $\alpha_{\nu}=2-\delta_{\nu, 0}$. The degeneracy factor $g_{i}=3$ for quarks and $g_{i}=1$ for leptons, and the Fermi energy for quarks and leptons is

$$
\mu_{i}^{*}=\sqrt{k_{F, \nu}^{i}{ }^{2}+s_{i}(\nu, B)^{2}}
$$

with $k_{F, \nu}^{i}$ being the Fermi momentum and $s_{i}(\nu, B)=$ $\sqrt{m_{i}^{2}+2 \nu\left|q_{i}\right| B}$. The highest Landau level is defined as

$$
\nu_{\max }^{i} \equiv \operatorname{int}\left[\frac{\mu_{i}^{* 2}-m_{i}^{2}}{2\left|q_{i}\right| B}\right]
$$

where int $[. .$.$] is the integer function. The total energy$ density of the system is thus obtained as

$$
\begin{aligned}
\mathcal{E}_{\text {tot }}= & \Omega+\sum_{i=u, d, s, l} \mu_{i}^{*} n_{i} \\
= & \sum_{i=u, d, s, l} \sum_{\nu=0}^{\nu_{\max }^{i}} \frac{g_{i}\left(\left|q_{i}\right| B\right)}{4 \pi^{2}} \alpha_{\nu} \int_{-\infty}^{\infty} \mathrm{d} p_{z} E_{p, i}+\frac{B^{2}}{2} \\
= & \sum_{i=u, d, s, l} \sum_{\nu=0}^{\nu_{\max }^{i}} \frac{g_{i}\left(\left|q_{i}\right| B\right)}{4 \pi^{2}} \alpha_{\nu}\left\{\mu_{i}^{*} \sqrt{\mu_{i}^{* 2}-s_{i}(\nu, B)^{2}}\right. \\
& \left.+s_{i}(\nu, B)^{2} \ln \left[\frac{\mu_{i}^{*}+\sqrt{\mu_{i}^{* 2}-s_{i}(\nu, B)^{2}}}{s_{i}(\nu, B)}\right]\right\}+\frac{B^{2}}{2},
\end{aligned}
$$

where the term $B^{2} / 2$ comes from the magnetic field contribution and $n_{i}$ is the number density of quarks and leptons given by

$$
n_{i}=\frac{g_{i}\left|q_{i}\right| B}{2 \pi^{2}} \sum_{\nu=0}^{\nu_{\max }^{i}}\left(2-\delta_{\nu}, 0\right) k_{F, \nu}^{i}
$$

We note that for the cases we consider in this work, muons will not appear in SQM due to the small chemical potential of electrons. The electric charge neutrality condition of SQM can thus be written as

$$
\frac{2}{3} n_{u}=\frac{1}{3} n_{d}+\frac{1}{3} n_{s}+n_{e}
$$

For SQM, we assume it is neutrino free and the $\beta$ equilibrium condition in SQM can then be expressed as

$$
\mu_{u}+\mu_{e}=\mu_{d}=\mu_{s}
$$

where $\mu_{i}\left(i=u, d, s\right.$ and $\left.e^{-}\right)$represents the particle chemical potential. For quarks, the chemical potential in SQM can be obtained as

$$
\mu_{i}=\frac{d \mathcal{E}_{t o t}}{d n_{i}}=\mu_{i}^{*}+\sum_{j=u, d, s} \frac{\partial \Omega_{j}}{\partial m_{j}} \frac{\partial m_{j}}{\partial n_{i}} .
$$

One can see from Eq. (12) that, owing to the density dependence of the equivalent quark mass, there are additional terms in the chemical potential compared to the case of free Fermi gas. Therefore, the chemical potential of $u, d$ and $s$ quarks in SQM can be obtained, respectively, as

$\mu_{u}=\mu_{u}^{*}+D_{I} n_{B}^{\alpha} e^{-\beta n_{B}}\left[\frac{\partial \Omega_{u}}{\partial m_{u}}-\frac{\partial \Omega_{d}}{\partial m_{d}}\right] \frac{6 n_{d}}{\left(n_{u}+n_{d}\right)^{2}}+\mu_{d e n}$,

$\mu_{d}=\mu_{d}^{*}+D_{I} n_{B}^{\alpha} e^{-\beta n_{B}}\left[\frac{\partial \Omega_{d}}{\partial m_{d}}-\frac{\partial \Omega_{u}}{\partial m_{u}}\right] \frac{6 n_{u}}{\left(n_{u}+n_{d}\right)^{2}}+\mu_{d e n}$,

$\mu_{s}=\mu_{s}^{*}+\mu_{d e n}$, 
with

$$
\begin{aligned}
\frac{\partial \Omega_{f}}{\partial m_{f}}= & \frac{3}{2 \pi^{2}} \sum_{\nu=0}^{\nu_{\max }^{f}} \alpha_{\nu}\left(\left|q_{f}\right| B m_{f}\right) \times \\
& \ln \left[\frac{k_{F, \nu}^{f}+\sqrt{k_{F, \nu}^{f}{ }^{2}+2 \nu\left|q_{f}\right| B+m_{f}^{2}}}{\sqrt{2 \nu\left|q_{f}\right| B+m_{f}^{2}}}\right],
\end{aligned}
$$

and

$$
\begin{aligned}
\mu_{\text {den }}= & \frac{1}{3} \sum_{j=u, d, s} \frac{3}{2 \pi^{2}} \sum_{\nu=0}^{\nu_{\max }^{j}} \alpha_{\nu}\left(\left|q_{j}\right| B\right) m_{j} \times \\
& \ln \left[\frac{k_{F, \nu}^{j}+\sqrt{k_{F, \nu}^{j}+2}+2 \nu\left|q_{j}\right| B+m_{j}^{2}}{\sqrt{2 \nu\left|q_{j}\right| B+m_{j}^{2}}}\right] \times \\
& \left.\left\{-\frac{z D}{n_{B}^{(1+z)}}-\tau_{j} D_{I} \delta\left[\alpha n_{B}^{\alpha-1}-\beta n_{B}^{\alpha}\right] e^{-\beta n_{B}}\right\} 1.7\right)
\end{aligned}
$$

The chemical potential of leptons can be written as

$$
\mu_{l}=\sqrt{k_{F, \nu}^{l}{ }^{2}+s_{l}(\nu, B)^{2}} .
$$

For SQM under a constant magnetic field, the $\mathcal{O}(3)$ rotational symmetry is broken and the pressure of the system becomes anisotropic, i.e., it is split into the longitudinal pressure $P_{\|}$which is parallel to the magnetic field and the transverse pressure $P_{\perp}$ which is perpendicular to the magnetic field. The expressions of $P_{\|}$and $P_{\perp}$ for a magnetized fermion system can be written as [43]

$$
\begin{aligned}
P_{\|} & =\sum_{i} \mu_{i} n_{i}-\mathcal{E}_{t o t}, \\
P_{\perp} & =\sum_{i} \mu_{i} n_{i}-\mathcal{E}_{t o t}+B^{2}-M B
\end{aligned}
$$

where $M$ is the system magnetization. It is interesting to see that the longitudinal pressure $P_{\|}$satisfies the Hugenholtz-Van Hove (HVH) theorem [68] while the transverse pressure $P_{\perp}$ has extra contributions from the magnetic field. This feature will lead to the fact that the zero-pressure point density coincides with the density at the minimum of the energy per baryon for $P_{\|}$but not for $P_{\perp}$, as will be shown later.

In the CIDDM model, the longitudinal and transverse pressures of the system under a constant magnetic field can thus be expressed, respectively, as

$$
\begin{aligned}
P_{\|} & =-\sum_{i=u, d, s, l} \Omega_{i}+\sum_{i, j=u, d, s} \frac{\partial \Omega_{j}}{\partial m_{j}} \frac{\partial m_{j}}{\partial n_{i}} n_{i}-\frac{B^{2}}{2}, \quad(21) \\
P_{\perp} & =-\sum_{i=u, d, s, l} \Omega_{i}+\sum_{i, j=u, d, s} \frac{\partial \Omega_{j}}{\partial m_{j}} \frac{\partial m_{j}}{\partial n_{i}} n_{i}+\frac{B^{2}}{2}-M B,
\end{aligned}
$$

where the system magnetization $M$ is given by

$$
M=-\partial \Omega / \partial B=\sum_{i=u, d, s, l} M_{i}
$$

with

$M_{i}=-\frac{g_{i}\left|q_{i}\right|}{2 \pi^{2}} \sum_{\nu=0}^{\nu_{\max }^{i}}\left(2-\delta_{\nu 0}\right) \int_{0}^{k_{F, \nu}^{i}}\left[\frac{\nu\left|q_{i}\right| B}{\epsilon_{\nu}^{i}}+\epsilon_{\nu}^{i}-\mu_{i}^{*}\right] d k_{z}$,

and $\epsilon_{\nu}^{i}=\sqrt{k_{z}^{2}+m_{i}^{2}+2 \nu\left|q_{i}\right| B}$. It should be emphasized that the longitudinal and transverse pressures in Eqs. (21) and (22) include the contributions from the magnetic fields. In particular, one can see that the magnetic energy density term $B^{2} / 2$ contributes oppositely to the longitudinal and transverse pressures under a constant magnetic field, and it decreases the former while increases the latter, which will lead to a tremendous difference between the longitudinal and transverse pressure when the magnetic field is very strong.

\section{Density-dependent magnetic fields in quark stars}

It is generally believed that the magnetic field strength in the core of compact stars should be much larger than that at the surface, and a density-dependent magnetic field profile is usually introduced to describe this behavior for the spatial distribution of the magnetic field strength in the compact stars [47]. In the present work, we use the following popular parametrization for the densitydependent magnetic field profile in QSs 47 51]

$$
B=B_{\text {surf }}+B_{0}\left[1-\exp \left(-\beta_{0}\left(n_{B} / n_{0}\right)^{\gamma}\right)\right],
$$

where $B_{\text {surf }}$ is the magnetic field strength at the surface of compact stars and its value is fixed at $B_{\text {surf }}=10^{15} \mathrm{G}$ in this work, $n_{0}=0.16 \mathrm{fm}^{-3}$ is the normal nuclear matter density, $B_{0}$ is a parameter with dimension of $B, \beta_{0}$ and $\gamma$ are two dimensionless parameters that control how exactly the magnetic field strength decays from the center to the surface.

Besides the spatial distribution of the magnetic field strength in the compact stars, the orientation of the magnetic fields is also expected to be important for the structure of the compact stars since the pressure (including the contribution from the magnetic fields) may become significantly anisotropic under strong magnetic fields. Consequently, the gravitational field in magnetized stars is no longer spherically symmetric due to the pressure anisotropy and the well-known Tolman-OppenheimerVolkoff (TOV) equations [69] generally cannot be applied to calculate the structure of the magnetized compact stars since they are only valid for spherically symmetric compact stars.

Since there is no empirical information on the magnetic field orientation inside the compact stars, in the 
present work, we assume two extremely special cases for the orientation of the magnetic fields inside the compact stars: one is that the local magnetic fields are along the radial direction (denoted as "radial orientation"), and the other is that the local magnetic fields are perpendicular to the radial direction but randomly oriented in the plane perpendicular to the radial direction (denoted as "transverse orientation"). It should be mentioned that the magnetic fields pass through the centra of the spherical compact stars for the radial orientation. In these two extreme cases, the pressure distribution inside the static compact stars can be considered to be spherically symmetric and thus the gravitational field as well as the static compact stars are spherically symmetric too. For these two extreme cases for the orientation of the magnetic fields, one thus can calculate the structure of the static magnetized compact stars by solving the following TOV equations:

$$
\begin{aligned}
\frac{d M(r)}{d r}= & 4 \pi r^{2} \epsilon(r) \\
\frac{d p(r)}{d r}= & -\frac{G \epsilon(r) M(r)}{r^{2}}\left[1+\frac{p(r)}{\epsilon(r)}\right]\left[1+\frac{4 \pi p(r) r^{3}}{M(r)}\right] \times \\
& {\left[1-\frac{2 G M(r)}{r}\right]^{-1} }
\end{aligned}
$$

where $M(r)$ is the total mass inside the sphere of radius $r, \epsilon(r)$ is the corresponding energy density (including the magnetic field contribution), $p(r)$ is the corresponding (radial) pressure (including the magnetic field contribution), and $G$ is Newton's gravitational constant.

We would like to point out that the radial and transverse orientations have been assumed to be inside the compact stars and the magnetic field orientation outside the compact stars or close the surface of the compact stars may change and become nonspherically symmetric. On the other hand, it should be mentioned that around the surface of compact stars, the magnetic field strength relatively is quite weak $\left(\sim 10^{15} \mathrm{G}\right)$ and the pressure is very small, and thus the magnetic fields around the surface is not important for the structure of QSs. For more general cases of magnetic field orientations and/or distributions in the magnetized compact stars where the spherical symmetry is broken, Einstein field equations should be solved self-consistently to calculate the structure of the compact stars, and this is beyond the scope of the present work and yet to be constructed.

\section{RESULTS AND DISCUSSIONS}

\section{A. EOS of SQM under a constant magnetic field}

We first present the results for the properties of SQM under a constant magnetic field. Using the CIDDM model with DI-85 $(\mathrm{z}=1.8)$, we show in Fig. 1 the energy per baryon and the corresponding longitudinal and

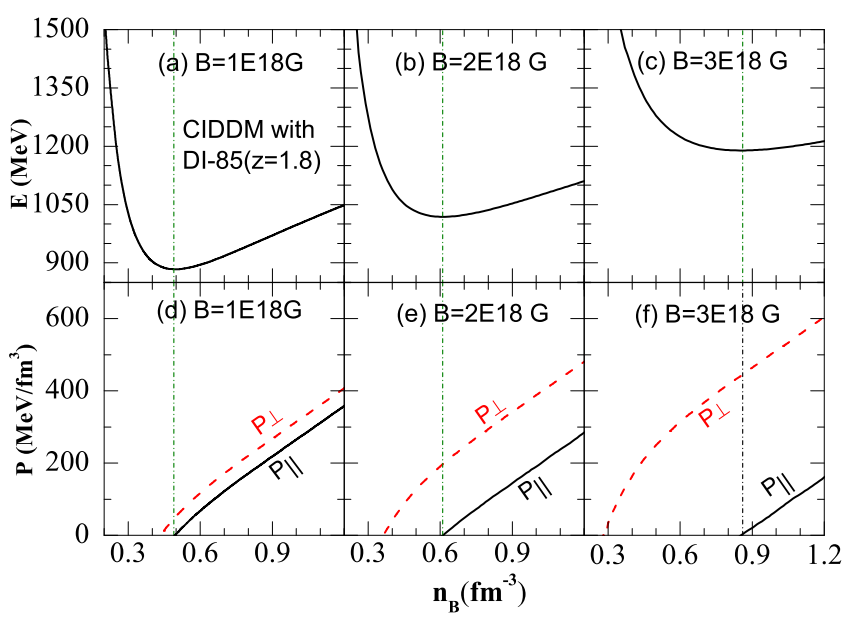

FIG. 1: (Color online) Energy per baryon and the corresponding longitudinal and transverse pressures as functions of the baryon density for SQM under constant magnetic fields with strengthes of $B=1 \times 10^{18} \mathrm{G}, 2 \times 10^{18} \mathrm{G}$ and $3 \times 10^{18} \mathrm{G}$ within the CIDDM model with DI-85 ( $\mathrm{z}=1.8)$.

transverse pressures as functions of the baryon density for SQM under constant magnetic fields with three strengthes of $B=1 \times 10^{18} \mathrm{G}, 2 \times 10^{18} \mathrm{G}$ and $3 \times 10^{18}$ $G$. One can see that for all the three values of the magnetic field strength $B$, the density at the minimum of energy per baryon is exactly equal to the zero point density of the longitudinal pressure $P_{\|}$, which is consistent with HVH theorem (as shown in Eq. (19)) and the thermodynamic self-consistency as in the case without magnetic fields [38]. Furthermore, it is seen that the density at the minimum of energy per baryon increases with the magnetic field strength, i.e., it varies from $0.49 \mathrm{fm}^{-3}$ to $0.61 \mathrm{fm}^{-3}$ and then to $0.86 \mathrm{fm}^{-3}$ when $B$ changes from $1 \times 10^{18} \mathrm{G}$ to $2 \times 10^{18} \mathrm{G}$ and then to $3 \times 10^{18} \mathrm{G}$.

In addition, one can also see from Fig. 1 that at a fixed density, the transverse pressure $P_{\perp}$ increases while the longitudinal pressure $P_{\|}$decreases with the increment of the magnetic field strength $B$, leading to a clear splitting between $P_{\perp}$ and $P_{\|}$under the constant magnetic fields. This pressure splitting (i.e., anisotropy) rapidly increases with $B$. The pressure anisotropy under strong magnetic fields is due to the additional terms of $B^{2}$ and magnetization of the system appeared in $P_{\perp}$ as shown in Eqs. (19) and (20).

In order to quantitatively describe the pressure anisotropy under strong magnetic fields, one can define a normalized pressure splitting factor as

$$
\delta_{p}=\frac{P_{\perp}-P_{\| \mid}}{\left(P_{\perp}+P_{\| \mid}\right) / 2} .
$$

From this definition, one has $\delta_{p}=0$ if there is no splitting between $P_{\perp}$ and $P_{\|}$, and $\delta_{p}=2$ for the extremely anisotropic case with $P_{\|}=0$. Shown in Fig. 2 are the transverse and longitudinal pressures together with the pressure splitting factor $\delta_{p}$ as functions of the magnetic 


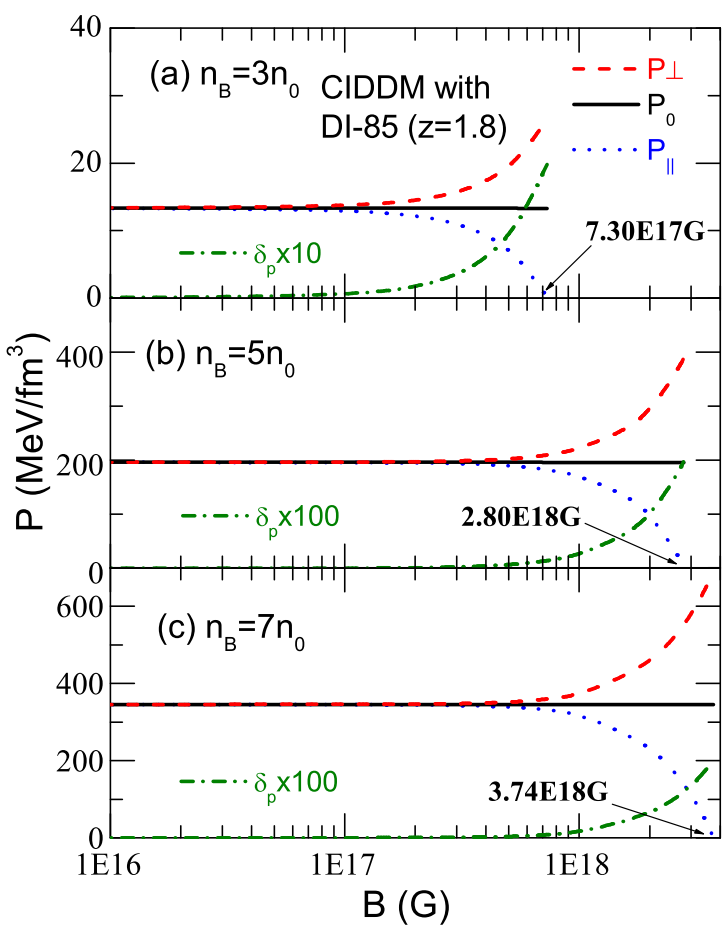

FIG. 2: (Color online) Transverse and longitudinal pressures together with the pressure splitting factor $\delta_{p}$ as functions of the magnetic field strength $B$ for SQM at baryon number densities of $n_{B}=3 n_{0}, 5 n_{0}$, and $7 n_{0}$ within the CIDDM model with DI-85 ( $\mathrm{z}=1.8)$. The corresponding pressures at $B=0$ $\left(P_{0}\right)$ are also included for comparison.

field strength $B$ for SQM at three baryon number densities of $n_{B}=3 n_{0}, 5 n_{0}$, and $7 n_{0}$ within the CIDDM model with DI-85 $(\mathrm{z}=1.8)$. For comparison, the corresponding pressures at $B=0$ are also included Fig. 2. We would like to point out that the central baryon density in QSs is roughly around $7 n_{0}$, and $n_{B}=3 n_{0}$ and $5 n_{0}$ are two typical values of baryon density in QSs.

One can see from Fig. 2 that, when the magnetic field strength is larger than a certain value of $B_{m}$ below which the magnetic field effects on the pressure are essentially negligible (with $\delta_{p} \leq 5 \%$ ), the transverse pressure $P_{\perp}$ increases rapidly while the longitudinal pressure $P_{\|}$decreases rapidly with increment of $B$, leading to a rapid enhancement of $\delta_{p}$. When the magnetic field strength $B$ further increases and reaches a critical value of $B_{c}$, the $P_{\|}$ becomes to zero (and thus $\delta_{p}=2$ ). When the magnetic field strength is larger than $B_{c}$, the $P_{\|}$becomes negative and thus the system becomes unstable. Therefore, $B_{c}$ is the largest magnetic field strength that a stable SQM in QSs can have. In addition, it is seen from Fig. 2 that the values of $B_{m}$ and $B_{c}$ depend on the baryon density, and particularly we have $B_{m} \approx 1.5 \times 10^{17} \mathrm{G}$ and $B_{c} \approx 7.30 \times 10^{17} \mathrm{G}$ for $n_{B}=3 n_{0}, B_{m} \approx 4.5 \times 10^{17} \mathrm{G}$ and $B_{c} \approx 2.80 \times 10^{18} \mathrm{G}$ for $n_{B}=5 n_{0}$, and $B_{m} \approx 6.0 \times 10^{17} \mathrm{G}$ and $B_{c} \approx 3.74 \times 10^{18} \mathrm{G}$ for $n_{B}=7 n_{0}$. For a magnetized compact star, it is thus important to ensure $B<B_{c}$ for

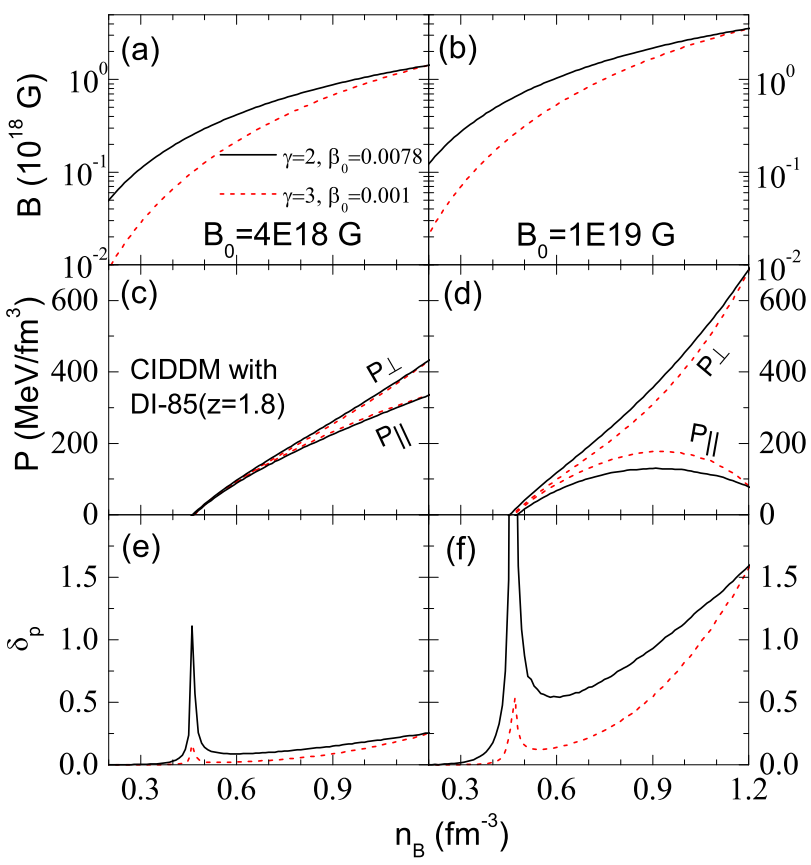

FIG. 3: (Color online) Baryon density dependence of the magnetic field strength $B$, longitudinal and transverse pressures as well as the pressure splitting factor $\delta_{p}$ for SQM in QSs using the slow B-profile (solid lines) and fast B-profile (dashed lines) within the CIDDM model with DI-85 ( $\mathrm{z}=1.8) . \quad B_{0}=4 \times 10^{18}$ $\mathrm{G}$ (left panels) and $1 \times 10^{19} \mathrm{G}$ (right panels) are considered.

all matter inside the compact stars.

\section{B. Quark stars under density dependent magnetic fields}

As mentioned earlier, the magnetic field strength is generally believed to be varied inside the magnetized compact stars, and a density-dependent magnetic field profile of Eq. (25) is usually introduced to mimic the magnetic field strength distribution inside the stars. Due to our poor knowledge on the magnetic field strength distribution inside the stars, we consider in this work two sets of values for the dimensionless parameters $\beta_{0}$ and $\gamma$, i.e., the fast-varied magnetic field profile with $\gamma=3$ and $\beta_{0}=0.001$ (denoted as "fast B-profile") and the slowvaried magnetic field profile with $\gamma=2$ and $\beta_{0}=0.0078$ (denoted as "slow B-profile"). Using these two different magnetic field profiles, we show in Fig. 3 the density dependence of the magnetic field strength $B$, the longitudinal and transverse pressures as well as the corresponding pressure splitting factor $\delta_{p}$ for SQM using $B_{0}=4 \times 10^{18}$ $\mathrm{G}$ and $B_{0}=1 \times 10^{19} \mathrm{G}$ within the CIDDM model with DI-85 ( $\mathrm{z}=1.8)$.

From Fig. 3, one can see that the fast B-profile gives a stronger density dependence of magnetic field strength, i.e., a faster decay for the magnetic field strength from high densities (e.g., the core of compact stars) to low 


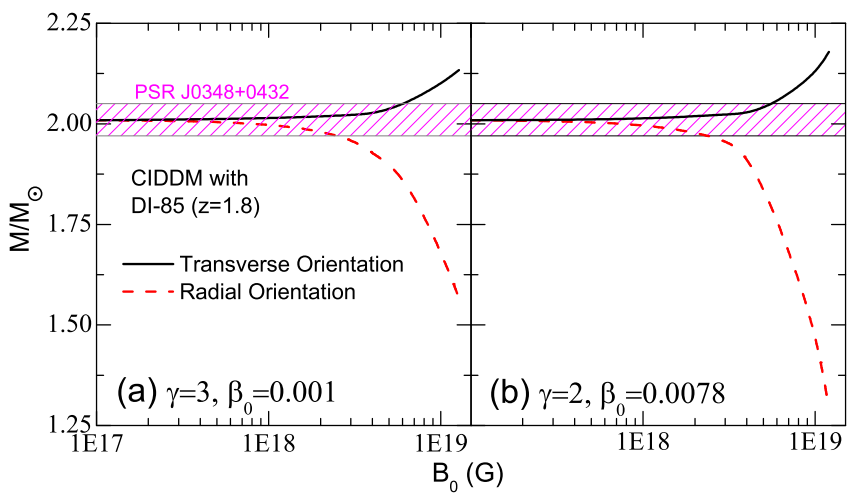

FIG. 4: (Color online) Maximum mass of static QSs using the transverse and radial orientations of the magnetic fields as a function of $B_{0}$ with the fast B-profile (a) and the slow Bprofile (b) within the CIDDM model with DI- $85(\mathrm{z}=1.8)$. The shaded band represents the pulsar mass of $2.01 \pm 0.04 M_{\odot}$ from PSR J0348+0432 [62].

densities (e.g., the surface of compact stars), than the slow B-profile as expected. For the smaller value of $B_{0}$ $\left(B_{0}=4 \times 10^{18} \mathrm{G}\right)$, one can see that $P_{\perp}$ is larger than $P_{\|}$ at higher densities and then they approach to zero almost at the same density of about $0.46 \mathrm{fm}^{-3}$. For this smaller value of $B_{0}$, the pressure splitting between $P_{\perp}$ and $P_{\|}$is not so big and we have $\delta_{p}=0.25$ (i.e., $P_{\|} / P_{\perp}=0.78$ ) at $1.2 \mathrm{fm}^{-3}$. The peak of $\delta_{p}$ around $0.46 \mathrm{fm}^{-3}$ is due to the vanishing of $P_{\|}$there, which corresponds to the surface of QSs. In addition, it is seen that the difference between the EOSs of SQM with the fast B-profile and the slow B-profile is small for $B_{0}=4 \times 10^{18} \mathrm{G}$. On the other hand, for $B_{0}=1 \times 10^{19} \mathrm{G}$, one can see that $P_{\perp}$ is significantly larger than $P_{\|}$at higher densities, and while $P_{\perp}$ always increases with $n_{B}, P_{\|}$decreases with $n_{B}$ when $n_{B} \geq 0.9 \mathrm{fm}^{-3}$, leading to a very big pressure splitting between $P_{\perp}$ and $P_{\|}$at higher densities, i.e., $\delta_{p}=1.6$ (corresponding to $P_{\|} / P_{\perp}=1 / 9$ ) at $1.2 \mathrm{fm}^{-3}$. Therefore, the pressure could be strongly anisotropic in the core of QSs for $B_{0}=1 \times 10^{19} \mathrm{G}$. Furthermore, for the larger $B_{0}$ (i.e., $B_{0}=1 \times 10^{19} \mathrm{G}$ ), one can see from Fig. 3 that different B-profiles have obvious effects on $P_{\perp}$ and $P_{\|}$as well as their splitting.

The strong pressure anisotropy under strong magnetic fields implies the orientation of the magnetic fields in QSs should play an important role on the structure of QSs. Shown in Fig. 4 is the maximum mass of static QSs using the transverse and radial orientations of the magnetic fields as a function of $B_{0}$ with the fast B-profile and the slow B-profile within the CIDDM model with DI-85 $(\mathrm{z}=1.8)$. It is interesting to see that, while the maximum mass of static QSs increases with $B_{0}$ for the transverse orientation, it significantly decreases with $B_{0}$ for the radial orientation, especially when $B_{0}$ is larger than about $3 \times 10^{18} \mathrm{G}$. This orientation effect becomes more pronounced for the slow B-profile.

For the fast B-profile, one can see from Fig. 团(a) that the maximum mass of static QSs with the transverse (radial) orientation can reach about $2.13 M_{\odot}\left(1.57 M_{\odot}\right)$ at $B_{0} \approx 1.28 \times 10^{19} \mathrm{G}$ which corresponds to the upper limit of $B_{0}$ and further increasing $B_{0}$ would lead to negative $P_{\|}$in the core of the QSs. In order to see the effect of the magnetic field orientation on the maximum mass of QSs, we define the normalized mass asymmetry $\delta_{m}$ for the maximum QS mass as

$$
\delta_{m}=\frac{M_{\perp}-M_{\|}}{\left(M_{\perp}+M_{\|}\right) / 2},
$$

where $M_{\perp}\left(M_{\|}\right)$represents the maximum mass of QSs with transverse (radial) orientation. For the fast Bprofile, the largest mass asymmetry is found to be $\delta_{m}=$ $30 \%$ at $B_{0}=1.28 \times 10^{19} \mathrm{G}$ from Fig. 团(a).

In addition, for the slow B-profile, it is seen from Fig. (b) that the maximum mass of static QSs with the transverse (radial) orientation can reach about $2.18 M_{\odot}$ $\left(1.29 M_{\odot}\right)$ at $B_{0}=1.20 \times 10^{19} \mathrm{G}$ which corresponds to the upper limit of $B_{0}$ above which the negative $P_{\|}$can appear in the core of QSs, and the corresponding largest mass asymmetry is $\delta_{m}=51 \%$ at $B_{0}=1.20 \times 10^{19} \mathrm{G}$. Therefore, our results indicate that the maximum mass of magnetized QSs may depend on both the strength distribution and the orientation of the magnetic fields inside the stars.

It should be mentioned that the above results and discussions are based on a special interaction parameter set, i.e., DI-85 $(\mathrm{z}=1.8)$, in the CIDDM model. In order to check the robustness of our conclusions and see the effects of the isospin dependence of equivalent quark mass on the properties of SQM under strong magnetic fields, we have further investigated the case with $D_{I}=0$, i.e., the parameter set DI-0 ( $\mathrm{z}=1.8)$, for which we have $D_{I}=0$ (thus the parameters $\alpha$ and $\beta$ are not involved), $D=26.483 \mathrm{MeV} \cdot \mathrm{fm}^{-3 z}, z=1.8, m_{u 0}=m_{d 0}=5.5$ $\mathrm{MeV}$ and $m_{s 0}=80 \mathrm{MeV}$, and the maximum QS mass predicted by DI-0 $(\mathrm{z}=1.8)$ without including magnetic fields is $1.88 M_{\odot}$. We find that the parameter set DI$0(\mathrm{z}=1.8)$ generally predicts a softer EOS of SQM and smaller values of the maximum QS mass but almost the same magnetic field effects on the pressure anisotropy (pressure splitting factor $\delta_{p}$ ) and the QS mass asymmetry $\delta_{m}$, compared with the parameter set DI-85 $(\mathrm{z}=1.8)$. Due to the softening of the EOS of SQM with DI-0 $(z=1.8)$, the values of the critical magnetic field strength $B_{c}$ as shown in Fig. 2 decrease correspondingly and even disappear (e.g., at $n_{B}=3 n_{0}$ ). These features imply that our main conclusion about the magnetic field effects on the properties of QSs remains unchanged even if various interactions are used.

Furthermore, the high density quark matter might be in a color superconducting phase [70]. The possible quark color superconducting phases mainly include the two-flavor color superconductor (2SC) [19, 71, 72], the color-flavor-locked (CFL) phase 73 78], and the crystalline color superconductor (CCS) 79]. In the present work, for simplicity, we have not considered color super- 
conducting phases. In recent years, significant progress has been made to understand the magnetic field effects on the properties of quark color superconducting phases [43, 54, 80 83]. In particular, by modeling the quark confinement within an effective bag model, the EOS of the magnetic-color-flavor-locked (MCFL) phase and the corresponding QS structure under a constant magnetic field have been investigated in Ref. [54]. It is nice to see that the magnetic field effects on the pressure anisotropy and the QS mass obtained in Ref. [54] are quite similar with our present predictions based on the density dependent magnetic field strength in the CIDDM model. It will be interesting to see how exactly the quark color superconducting phases affect the properties of SQM and QSs under strong magnetic fields within the CIDDM model.

\section{CONCLUSIONS}

We have studied the properties of strange quark matter and quark stars under strong magnetic fields by using the confined isospin- and density-dependent mass model. The equation of state of strange quark matter under constant magnetic fields has been calculated selfconsistently and the pressure of the system is shown to be anisotropic along and perpendicular to the magnetic field direction with the former being generally larger than the latter. The pressure of the system may become significantly anisotropic when the magnetic field strength is very strong and thus the properties of magnetized quark stars may significantly depend on the magnetic field orientation inside the stars.

Using a density-dependent magnetic field profile to simulate the magnetic field strength distribution in a star, we have studied the properties of static spherical quark stars by considering two hypothetical cases for the orientation of the magnetic fields inside the stars, i.e., the radial orientation in which the local magnetic fields are along the radial direction and the transverse orientation in which the local magnetic fields are perpendicular to the radial direction but randomly oriented in the plane perpendicular to the radial direction. Based on these two extreme cases of the magnetic field orientation, we have demonstrated that the maximum mass of static magnetized quark stars may significantly depend on the magnetic field orientation inside the stars, and the magnetic fields with radial (transverse) orientation can significantly decrease (increase) the maximum mass of the quark stars. The maximum mass of static magnetized quark stars has also been found to depend on the details of the density-dependent magnetic field profile.

Therefore, our present results have shown that besides the strength distribution, the orientation of the magnetic fields inside the quark stars is also important for the properties of quark stars under strong magnetic fields. For a fixed density-dependent magnetic field profile, including the magnetic fields can either enhance or reduce the maximum mass of static magnetized quark stars, depending on the magnetic field orientation inside the stars.

\section{ACKNOWLEDGMENTS}

This work was supported in part by the NNSF of China under Grant Nos. 11275125 and 11135011, the Shanghai Rising-Star Program under grant No. 11QH1401100, the "Shu Guang" project supported by Shanghai Municipal Education Commission and Shanghai Education Development Foundation, the Program for Professor of Special Appointment (Eastern Scholar) at Shanghai Institutions of Higher Learning, the National Basic Research Program of China (973 Program) under Contract No. 2015CB856900, and the Science and Technology Commission of Shanghai Municipality (11DZ2260700).
[1] N.K. Glendenning, Compact Stars, 2nd edition, SpingerVerlag New York, Inc., 2000.

[2] F. Weber, Pulsars as Astrophyical Laboratories for $\mathrm{Nu}-$ clear and Particle Physics, IOP Publishing Ltd, London, UK, 1999.

[3] J.M. Lattimer and M. Prakash, Science 304, 536 (2004).

[4] A.W. Steiner, M. Prakash, J.M. Lattimer, and P.J. Ellis, Phys. Rep. 410, 325 (2005).

[5] I. Bombaci, I. Parenti, and I. Vidana, Astrophy. J. 614, 314 (2004).

[6] J. Staff, R. Ouyed, and M. Bagchi, Astrophy. J. 667, 340 (2007).

[7] M. Herzog and F.K. Röpke, Phys. Rev. D 84, 083002 (2011).

[8] M.A. Stephanov, K. Rajagopal, and E.V. Shuryak, Phys. Rev. Lett. 81, 4816 (1998).

[9] A.R. Bodmer, Phys. Rev. D 4, 1601 (1971).

[10] H. Terazawa, INS-Report 336, Univ. of Tokyo, (1979).
[11] E. Witten, Phys. Rev. D 30, 272 (1984).

[12] A. Chodos, R.L. Jaffe, K. Ohnson, C.B. Thorn, and V.F. Weisskopf, Phys. Rev. D 9, 3471 (1974).

[13] E. Farhi and R.L. Jaffe, Phys. Rev. D 30, 2379 (1984).

[14] C. Alcock, E. Farhi, and A. Olinto, Astrophy. J. 310, 261 (1986).

[15] M. Alford, M. Braby, M. Paris, and S. Reddy, Astrophy. J. 629, 969 (2005).

[16] F. Weber, Prog. Part. Nucl. Phys. 54, 193 (2005).

[17] P. Rehberg, S.P. Klevansky, and J. Hüfner, Phys. Rev. C 53, 410 (1996).

[18] M. Hanauske, L.M. Satarov, I.N. Mishustin, H. Stocker, and W. Greiner, Phys. Rev. D 64, 043005 (2001).

[19] S.B. Rüster and D.H. Rischke, Phys. Rev. D 69, 045011 (2004).

[20] D.P. Menezes, C. Providencia, and D.B. Melrose, J. Phys. G 32, 1081 (2006).

[21] B.A. Freedman and L.D. Mclerran, Phys. Rev. D 16, 
1169 (1977).

[22] E.S. Fraga, R.D. Pisarski, and J. Schaffner-Bielich, Phys. Rev. D 63, 121702(R) (2001).

[23] E.S. Fraga and P. Romatschke, Phys. Rev. D 71, 105014 (2005).

[24] A. Kurkela, P. Romatschke, and A. Vuorinen, Phys. Rev. D 81, 105021 (2010).

[25] C.D. Roberts and A.G. Williams, Prog. Part. Nucl. Phys. 33, 477 (1994) and references therein.

[26] H.S. Zong, L. Chang, F.Y. Hou, W.M. Sun, and Y.X. Liu, Phys. Rev. C 71, 015205 (2005).

[27] S.X. Qin, L. Chang, H. Chen, Y.X. Liu, and C.D. Roberts, Phys. Rev. Lett. 106, 172301 (2011).

[28] G.N. Fowler, S. Raha, and R.M. Weiner, Z. Phys. C 9, 271 (1981).

[29] S. Chakrabarty, S. Raha, and B. Sinha, Phys. Lett. B229, 112 (1989).

[30] S. Chakrabarty. Phys. Rev. D 43, 627 (1991); 48, 1409 (1993); 54, 1306 (1996).

[31] O.G. Benvenuto and G. Lugones, Phys. Rev. D 51, 1989 (1995).

[32] G.X. Peng, H.C. Chiang, J.J. Yang, L. Li, and B. Liu, Phys. Rev. C 61, 015201 (1999).

[33] G.X. Peng, H.C. Chiang, B.S. Zou, P.Z. Ning, and S.J. Luo, Phys. Rev. C 62, 025801 (2000).

[34] G.X. Peng, A. Li, and U. Lombardo, Phys. Rev. C 77, 065807 (2008).

[35] A. Li, G.X. Peng, and J.F. Lu, Research Astron. Astrophys. 11, 482 (2011).

[36] K. Schertler, C. Greiner, and M.H. Thoma, Nucl. Phys. A616, 659 (1997).

[37] K. Schertler, C. Greiner, P.K. Sahu, and M.H. Thoma, Nucl. Phys. A637, 451 (1998).

[38] P.C. Chu and L.W. Chen, Astrophys. J. 780, 135 (2014).

[39] L. Woltjer, Astrophys. J. 140, 1309 (1964).

[40] T.A. Mihara, Nature (London) 346, 250 (1990).

[41] G. Chanmugam, Annu. Rev. Astron. Astrophys. 30, 143 (1992).

[42] D. Lai, and S.L. Shapiro, Astrophys. J. 383, 745 (1991).

[43] E.J. Ferrer, V. de la Incera, J.P. Keith, I. Portillo and P.L. Springsteen, Phys. Rev. C 82, 065802 (2010); E.J. Ferrer and V. de la Incera, Lect. Notes Phys. 871, 399 (2013).

[44] A.A. Isayev and J. Yang, Phys. Rev. C 84, 065802 (2011).

[45] A.A. Isayev and J. Yang, Phys. lett. B707, 163 (2012).

[46] A.A. Isayev and J. Yang, J. Phys. G 40, 035105 (2013).

[47] D. Bandyopadhyay, S. Chakrabarty, and S. Pal, Phys. Rev. Lett. 79, 2176 (1997).

[48] D. Bandyopadhyay, S. Pal, and S. Chakrabarty, J. Phys. G 24, 1647 (1998).

[49] D.P. Menezes, M. Benghi Pinto, S.S. Avancini, and C. Providência, Phys. Rev. C 79, 035807 (2009); 80, 065805 (2009).

[50] C.Y. Ryu, K.S. Kim and M.K. Cheoun, Phys. Rev. C 82, 025804 (2010).

[51] C.Y. Ryu, M.K. Cheoun, T. Kajino, T. Maruyama, and G.J. Mathews, Astropart. Phys. 38, 25 (2012).

[52] A. Broderick, M. Prakash, and J.M. Lattimer, Astrophys. J. 537, 351 (2000).

[53] C.Y. Cardall, M. Prakash, and J.M. Lattimer, Astrophys.
J. 554, 322 (2001).

[54] L. Paulucci, E.J. Ferrer, V. de la Incera, and J. E. Horvath, Phys. Rev. D 83, 043009 (2011).

[55] V. Dexheimer, D P Menezes, and M. Strickland, J. Phys. G 41, 015203 (2014).

[56] R.H. Casali, L.B. Castro, and D.P. Menezes, Phys. Rev. C 89, 015805 (2014)

[57] J.X. Hou, G.X. Peng, C.J. Xia, and J.F. Xu, arXiv:1403.1143. (2014).

[58] M. Di Toro, A. Drago, T. Gaitanos, V. Greco, and A. Lavagno, Nucl. Phys. A775, 102 (2006).

[59] G. Pagliara and J. Schaffner-Bielich, Phys. Rev. D 81, 094024 (2010).

[60] M. Di Toro, V. Baran, M. Colonna, and V. Greco, J. Phys. G 37, 083101 (2010).

[61] G.Y. Shao, M. Colonna, M. Di Toro, B. Liu, and F. Matera, Phys. Rev. D 85, 114017 (2012).

[62] J. Antoniadis et al., Science 340, 6131 (2013).

[63] L.D. Landau and E.M. Lifshitz, Quantum Mechanics (1965).

[64] A. Rabhi, H Pais, P K Panda, and C. Providência, J. Phys. G 36, 115204 (2009).

[65] X.J. Wen, S.Z. Su, D.H. Yang, and G.X. Peng, Phys. Rev. D 86, 034006 (2012).

[66] X.J. Wen, Phys. Rev. D 88, 034031 (2013).

[67] R. C. Duncan, arXiv:astro-ph/0002442, (2000).

[68] N.M. Hugenholtz and L. Van Hove, Physica 24, 363 (1958).

[69] J.R. Oppenheimer and G.M. Volkoff, Phys. Rev. 33, 374 (1939).

[70] M. Alford and A. Schmitt, Rev. Mod. Phys. 80, 1455 (2013)

[71] M. Alford, K. Rajagopal, and F. Wilczek, Phys. Lett. B422, 247 (1998).

[72] S.B. Rüster, V. Werth, M. Buballa, I.A. Shovkovy, and D.H. Rischke, Phys. Rev. D 72, 034004 (2005).

[73] M. Alford, K. Rajagopal, and F. Wilczek, Nucl. Phys. B537, 443 (1999).

[74] K. Rajagopal and F. Wilczek, Phys. Rev. Lett. 86, 3492 (2001).

[75] G. Lugones and J.E. Horvath, Phys. Rev. D 66, 074017 (2002).

[76] B.K. Agrawal and S.K. Dhiman, Phys. Rev. D 79, 103006 (2009).

[77] M.G.B. de Avellar, J.E. Horvath, and L. Paulucci, Phys. Rev. D 84, 043004 (2011).

[78] L. Paulucci, E.J. Ferrer, J.E. Horvath, and V. de la Incera, J. Phys. G 40, 125202 (2013).

[79] M.G. Alford, J. Bowers, and K. Rajagopal, Phys. Rev. D 63, 074016 (2001).

[80] D. Blaschke, D.M. Sedrakian, and K.M. Shahabasyan, Astron. Astrophys. 350, L47 (1999).

[81] E.J. Ferrer, V. de la Incera, and C. Manuel, Phys. Rev. Lett. 95, 152002 (2005).

[82] E.J. Ferrer and V. de la Incera,Phys. Rev. D 76, 045011 (2007).

[83] E.J. Ferrer, V. de la Incera, I. Portillo, and M. Quiroz, Phys. Rev. D 89, 085034 (2014). 